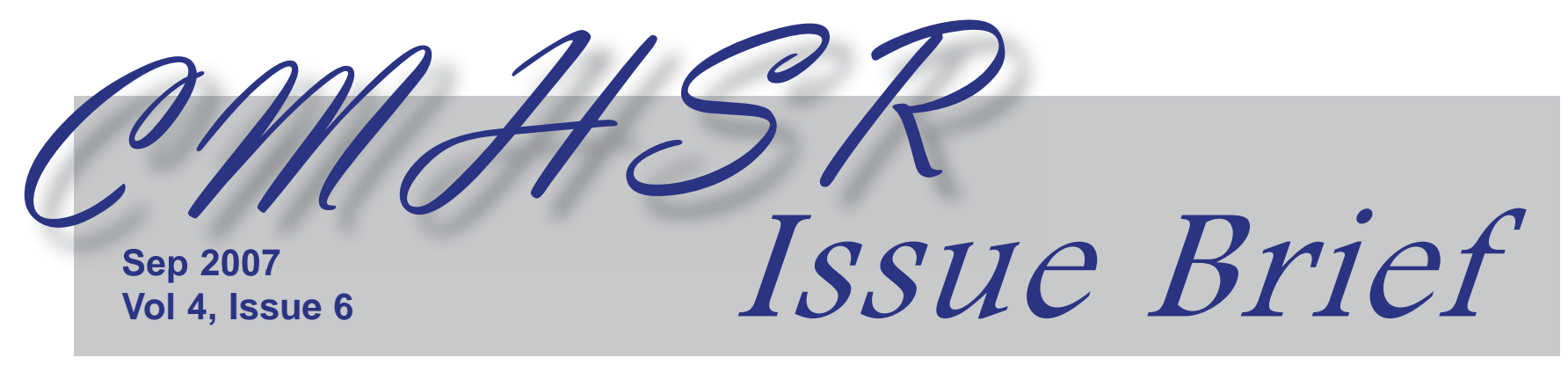

\title{
Does Routine Screening Matter? The Prenatal Emotional Health Screening Project (PEHS)
}

Jennifer Taub, Ph.D., Rebecca Lundquist, M.D., Kenneth Fletcher, Ph.D. \& Tara Zandi, B.S.

$\mathrm{P}$ ostpartum mood disorders (PMDs) affect new mothers and their ability to care for their newborns. ${ }^{1}$ An estimated $50-80 \%$ of all mothers experience "baby blues", 2 and 10-28\% of new mothers experience an episode of postpartum depression (PPD). . $^{2-4}$ Little research has examined the prevalence of PMDs other than PPD. With proper identification and referral, medication and psychotherapy have been shown to be effective treatments for PMDs. 5,6 However, in the absence of systematic screening, most PMDs are not detected. ${ }^{7}$

\section{Specific Aims}

With the aid of a grant from UMMS Commonwealth Medicine, the Prenatal Emotional Health Screening (PEHS) project was established to examine (1) the prevalence of PMDs other than depression (specifically, anxiety and PTSD), (2) biological risk factors which contribute to PMD development, and (3) the impact of systematic screening on PMD identification and referrals. The project screens for anxiety, depression and posttraumatic symptoms, and administers a 5-item Biological Risk Factor Questionnaire (BRFQ). The BRFQ assesses putative biological risk factors for PMD's such as personal and family history of depression, and mood changes related to shifts in women's hormones. The PEHS project also seeks to determine the overall feasibility of such routine screening in OB-GYN prenatal care settings.

Currently, the study is ongoing in two sites: (1) a community-based health center and (2) an OB-
GYN clinic housed in a University of Massachusetts Medical School affiliated teaching hospital. Both sites serve primarily Medicaid-eligible patients. Site 1 has a Healthy Start grant, ${ }^{8}$ which provides routine periodic depression screening and case management to most pregnant patients. Site 2 performs depression screening for about 1 in 5 patients, based on staff concerns about particular patients. Neither site screens for other mood disorders. Women who screen positive in any area are offered a free interview with a trained clinician to assess for further risk and to make appropriate referrals.

\section{Results}

The project has screened 139 women to date, $73 \%$ of whom were seen during prenatal visits.

PMD screening. Forty (29\%) of the women screened to date have screened positive for symptoms of depression, anxiety, or posttraumatic stress. Of these 40 , $85 \%$ were positive for depression, $48 \%$ had anxiety symptoms, and 53\% had PTSD symptoms. One in 5 women were comorbid with at least two of these areas, and 1 in 3 women were comorbid with all three areas. Of note, $15 \%$ of the 40 women screened positive for anxiety and/or PTSD, and did not screen positive for depression. Also of note, $90.5 \%$ of the women referred for treatment were being seen for prenatal rather than postnatal care.

Biological Risk Factor Questionnaire (BRFQ). The 5-item BRFQ totals correlated significantly with the depression (.35), anxiety (.36), and PTSD scale totals (.36). Women with a history of counseling 
for depression, a mother who suffered from depression, a history of premenstrual mood changes, or past PPD reported significantly higher scores for depression, anxiety, and symptoms of PTSD.

Referrals. After a one-session interview, about half $(\mathrm{n}=21)$ of the women who screened positive on at least one screening measure were referred for treatment. Most (90.5\%) of the 21 women referred for treatment had screened positive for depression, although $76 \%$ of them had screened positive for PTSD and 52\% for anxiety. If a woman originally screened positive for depression, there was a $54 \%$ chance she would be referred for treatment. If she screened positive for anxiety, there was a $58 \%$ chance of being referred, and if she screened positive for PTSD, there was a $76 \%$ chance of being referred (see figure 1). Note that there were participants who screened positive on one or more of the scales in the primary interview but refused the secondary screening and therefore the possibility of referral for treatment. Some of these women refused the secondary screen because they were already getting psychiatric treatment.

\section{Discussion and Future Directions}

Our preliminary results point to the high prevalence (15\%) of a range of mood problems among low income pregnant, pre- and postpartum women. A depression-only screen appears to identify an unexpectedly high proportion of women with potentially serious mood problems (regardless of the presence or absence of anxiety or PTSD). However, women who were comorbid for both depression and PTSD, were far more likely to be referred for treatment than those who were not. These results point to the utility of incorporating a brief depression screen into standard pre- and postnatal care (something already being done through Healthy Start initiatives).

Anxiety and PTSD screens can be somewhat labor intensive to administer and score in a busy OB-GYN setting, making them an impractical addition to practice as usual. In contrast, the 5-item $\mathrm{BRFQ}_{2}$ which asks women simple questions about their personal and family medical and psychiatric history, appears to have useful predictive value when assessing women for PMDs and may be simple for obstetrical providers to incorporate into standard care if it turns out to have predictive value. It could then be used by obstetrical providers to flag patients at risk for perinatal psychiatric illness and to concentrate intensive screening efforts on women who are most at risk.

Further analysis will help determine whether screening women while pregnant for symptoms and risk factors for psychiatric illness is useful. While current standards of practice call for screening only at the six week postpartum visits and not at other perinatal visits, initial project results suggest that many of the pregnant women with psychiatric needs can be captured in the prenatal period, increasing opportunities for intervention and prevention.

\section{Figure 1.}

Number of Women Screened in and Referred

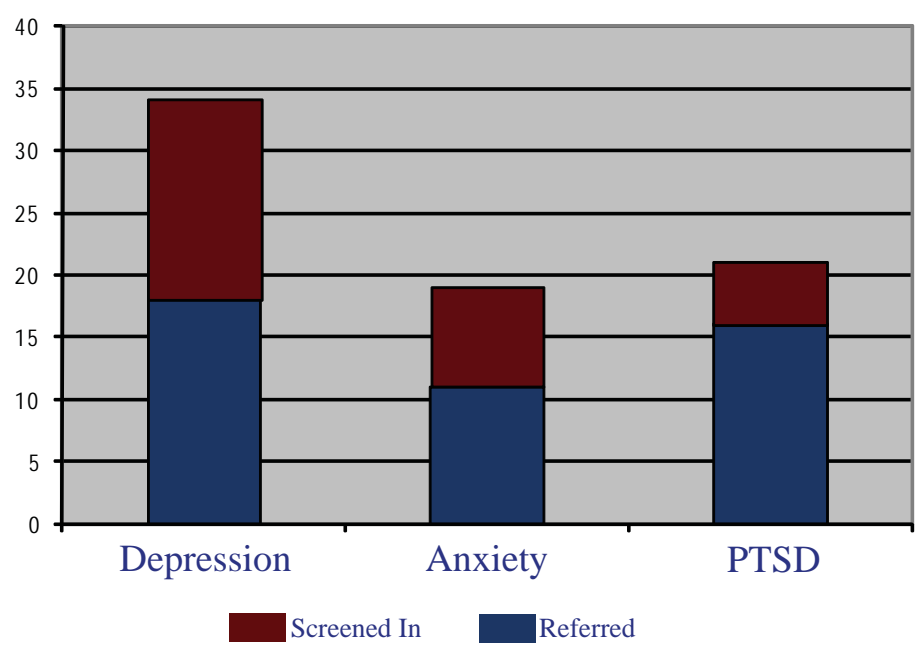

\section{References}

1. McLearn KT, Minkovitz CS, Strobino DM, Marks E, \& Hou W. (2006). Maternal depressive symptoms at 2 to 4 months post partum and early parenting practices. Archives of Pediatric E Adolescent Medicine, 160, 279-284. 2. Freeman MP, Wright R, Watchman M, et al. (2005). Postpartum depression assessments at well-baby visits: Screening feasibility, prevalence, and risk factors. Journal of Women's Health, 14, 929-35.

3. Georgiopoulos AM, Bryan TL, Yawn BP, Houston MS, Rummans TA, \& Therneau TM. (1999). Populationbased screening for postpartum depression. Obstetrics E Gynecology, 93, 653-7.

4. Stowe ZN \& Nemeroff CB. (1995). Women at risk for postpartum-onset major depression. American Journal of Obstetrics \& Gynecology, 173, 639-45.

5. Matthey S. (2004). Detection and treatment of postnatal depression (perinatal depression or anxiety). Current Opinion in Psychiatry, 17, 21-29.

6. Appleby L, Warner R, Whitton A, \& Faragher B. (1997). A controlled study of fluoxetine and cognitivebehavioural counselling in the treatment of postnatal depression.[see comment]. Bmj, 314, 932-6.

7. Matthey S. (2005). Assessing for psychosocial morbidity in pregnant women. Canadian Medical Association Journal, 173, 267-269.

8. http://www.healthystartassoc.org

\section{Visit us on-line at www.umassmed.edu/cmhsr}

